\title{
COLLISIONAL FOCUSING OF PARTICLES IN SPACE CAUSING JETSTREAMS
}

\author{
JAN TRULSEN \\ University of Troms $\phi$
}

Jetstreams probably played an important role at an intermediate stage of the formation of the solar system (Alfvén and Arrhenius, 1970). A jetstream is defined here as a collection of grains moving in neighboring elliptical orbits around a central gravitating body and with the dynamics modified by the action of complicated collision processes among the grains themselves.

Three main types of collisions will take place in such a stream. Hypervelocity impacts will lead to fragmentation of the grains involved. At lower impact velocities, the particles will retain their identities after the collision even if they might be deformed to some degree depending on impact velocity and internal structure of the grains. With still lower impact velocity, accretion can take place, the grains sticking together after collision to form larger grains. A common feature of these collision processes are that they will be partially inelastic. A certain fraction of the kinetic energy of the colliding particles will be spent on changing their internal structure. The internal kinetic energy in an isolated jetstream thus will tend to decrease with time. The mass spectrum of the grains also will vary during the lifetime of a stream, the probability for accretive processes increasing with time.

Qualitative arguments for the collisional focusing effect leading to the formation of streams have been given by Alfvén. The most important points in his argument are

(1) Two orbits after a collision will be more similar than before because of the loss of kinetic energy.

(2) Because of the central force field, a particle having collided with a stream is not easily lost from the stream. It will always return to the place where it last collided, thus making it subject to new collisions with particles in the stream.

As a first step toward a quantitative theory, the idealized situation of a jetstream of identical spherical grains will be studied. Collisions leading to fragmentation and accretion will be neglected together with the selfgravitational effect of the stream. The distribution of grains in the stream will be described by a distribution function $f(\mathbf{r}, \mathbf{v}, t)$ in the six-dimensional phase 
space. The first problem is to construct a kinetic equation describing the evolution of this distribution function due to the motion in the central gravitational field and to the mutual collisions.

The equations of motion of a single particle in a central gravitational force field are

$$
\dot{\mathbf{r}}=\mathbf{v} \quad \dot{\mathbf{v}}=-\frac{\mathbf{r}}{r^{3}}
$$

The orbits for bound particles are ellipses with the central body at one of the focal points. Time and length units have been chosen such that the orbital period in an ellipse with a semimajor axis equal to unity is $2 \pi$ time units.

The rate of change of the distribution function due to the motion in the gravitational field is

$$
\left(\frac{\partial f}{\partial t}\right)_{\text {motion }}=-\mathbf{v} \cdot \frac{\partial f}{\partial \mathbf{r}}+\frac{\mathbf{r}}{r^{3}} \cdot \frac{\partial f}{\partial \mathbf{v}} \equiv \Lambda f
$$

If the effect of collisions is described by a nonlinear operator $C$,

$$
\left(\frac{\partial f}{\partial t}\right)_{\text {coll }}=C[f, f]
$$

the complete kinetic equation takes the form

$$
\frac{\partial f}{\partial t}=\left(\frac{\partial f}{\partial t}\right)_{\text {motion }}+\left(\frac{\partial f}{\partial t}\right)_{\text {coll }}=\Lambda f+C[f, f]
$$

Already Boltzmann gave the form of the collision operator for the case of number-conserving elastic collisions (Chapman and Cowling, 1960). Because we are interested in partially inelastic collisions, a somewhat modified expression is needed.

\section{DERIVATION OF COLLISION OPERATOR}

The details of a collision process are described in figure 1. A collision takes place as soon as the distance between the centers of two particles is equal to their diameter $D$. The direction between the centers at impact is given by the impact vector $\mathbf{k}$ which is a unit vector. Particles 1 and 2 have velocities $\mathbf{v}_{1 b}$ and $\mathbf{v}_{2 b}$ before the collision. Afterward, the corresponding velocities are $\mathbf{v}_{1 a}$ and $\mathrm{v}_{2 a}$. 


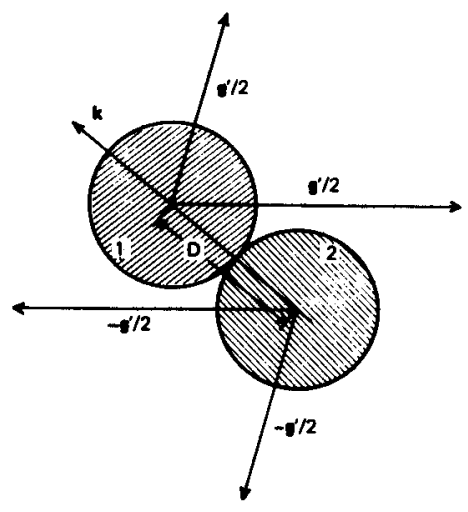

Figure 1.-Details of a collision event. $k$ is impact vector, $g$ and $g^{\prime}$ are relative velocities before and after the impact, respectively.

Conservation of momentum requires that

$$
\mathrm{v}_{1 b}+\mathrm{v}_{2 b}=\mathrm{v}_{1 a}+\mathrm{v}_{2 a}
$$

Kinetic energy is not conserved. To simplify the problem, we do not care what happens to this lost energy by simply prescribing the amount lost in a given collision by the relation

$$
\mathbf{v}_{1 b}{ }^{2}+v_{2 b}{ }^{2}+1 / 2\left[(\beta-1)^{2}-1\right]\left[\left(v_{1 b}-v_{2 b}\right) \cdot k\right]^{2}=v_{1 a}{ }^{2}+v_{2 a}{ }^{2}
$$

Here $\beta$ is a restitution parameter describing varying degrees of inelasticity. It is allowed to take any value in the range $(1,2)$. The upper limit, $\beta=2$, corresponds to elastic collisions. For the lower limit, $\beta=1$, a head-on collision will be completely inelastic. The amount of energy lost decreases as the collision takes place more and more off axis, and in the limit of a grazing collision there is no energy loss.

Proceeding in a manner similar to the derivation of the classical Boltzmann operator, the following form of the collision operator can be derived from equations (5) and (6):

$$
\begin{gathered}
C[f, f]=D^{2} \int d \mathbf{v}^{\prime} \int\left(\mathbf{v}^{\prime}-\mathbf{v}\right) \cdot \mathbf{k}\left[\frac{f(\overline{\mathbf{v}}) \cdot f\left(\overline{\mathbf{v}}^{\prime}\right)}{(\beta-1)^{2}}-f(\mathbf{v}) \cdot f\left(\mathbf{v}^{\prime}\right)\right] d \mathbf{k} \\
\left(\mathbf{v}^{\prime}-\mathbf{v}\right) \cdot \mathbf{k}>0
\end{gathered}
$$


Here the explicit $\mathbf{r}$ and $t$ dependence of the distribution function was suppressed and

$$
\begin{aligned}
& \overline{\mathbf{v}}=\mathbf{v}+1 / 2 \frac{\beta}{\beta-1}\left(\mathbf{v}^{\prime}-\mathbf{v}\right) \cdot \mathbf{k k} \\
& \overline{\mathbf{v}}^{\prime}=\mathbf{v}^{\prime}-1 / 2 \frac{\beta}{\beta-1}\left(\mathbf{v}^{\prime}-\mathbf{v}\right) \cdot \mathbf{k k}
\end{aligned}
$$

The operator (eq. (7)) reduces to the Boltzmann operator for the case $\beta=2$. The apparent singularity of equation (7) as the extreme case $\beta=1$ is approached is not real. For the product $f(\bar{v}) f\left(\bar{v}^{\prime}\right)$ to be nonvanishing, both $\nabla$ and $\overrightarrow{\mathbf{v}}^{\prime}$ must lie inside the support of $f$ in velocity space. This requires the quantity $\left(v^{\prime}-\mathbf{v}\right) \cdot \mathbf{k}$ to be of order $\beta-1$. Thus we get one factor $\beta-1$ from the volume element in velocity space and one from the factor $\left(\mathbf{v}^{\prime}-\mathbf{v}\right) \cdot \mathbf{k}$ already present in the integrand, making the operator (eq. (7)) tend toward a finite limit as $\beta \rightarrow 1$.

Entropy arguments through Boltzmann's $H$ theorem play an important role in the discussion of the classical Boltzmann equation. With elastic collisions, the entropy source function is always positive except for local thermodynamic equilibrium (LTE) distributions where it vanishes. For the motion in a central gravitational force field with a nonvanishing total angular momentum, however, it is not possible to construct a time-independent, physically relevant state from LTE distributions. For a physically relevant situation, the average flow velocity should increase with decreasing distance from the central body if all the grains are moving in the prograde direction. If, on the other hand, an LTE distribution function is substituted into the time-independent kinetic equation, the only allowed average velocity goes as $U=r \times \omega$ where $\boldsymbol{\omega}$ is a constant vector; that is, a fixed body rotation with a velocity increasing with increasing distance from the center (Chapman and Cowling, 1960). This result does not imply, however, that the system would not evolve asymptotically toward a time-independent final state. An additional possibility is that the collisions tend to make themselves less important by making the individual orbits more and more parallel, the whole system evolving toward a Saturnian ring configuration.

For the case of inelastic collisions, we no longer have a description of a closed system. We do not have a description of what happens to the lost kinetic energy. No extension of the $H$ theorem has been given for this case. In a way, the kinetic energy function plays the role of Boltzmann's $H$ function, giving the allowed direction of evolution of the system. Again, the only timeindependent state of the system is the singular state consisting of parallel individual orbits only.

\section{THE INITIAL RATE OF CHANGE PROBLEM}

The most usual way of extracting information about a system by linearizing the equations of motion around a time-independent equilibrium state is hardly 
of interest here for two reasons. We have already seen that this state is rather singular. Further, accretion would probably play a crucial role long before this state is approached. A complete analytic study of the dynamics seems out of the question at the present time. A more modest undertaking is to study an initial rate of change problem. How will the system start to change from a prescribed initial state $F(\mathbf{r}, \mathbf{v})$ ?

The crucial point now is to choose initial states that make it possible to extract interesting properties of the system. Because we are interested in the influence of collisions on the dynamics, $F(\mathbf{r}, \mathbf{v})$ should be chosen to be a time-independent solution of the collisionless kinetic equation; that is, $F$ should satisfy $\Lambda F=0$. Any function depending on $\mathbf{r}$ and $\mathbf{v}$ through only time-independent constants of motion for a central gravitational force field satisfies this requirement. Such constants are (Danby, 1962)

$$
\begin{aligned}
& a=\frac{r}{2-r v^{2}} \\
& L=r \times v \\
& P=-(r \times v) \times v-\frac{r}{r}
\end{aligned}
$$

being the semimajor axis, the angular momentum vector, and the perihelion vector, respectively. Only five of these constants are independent because the following two relations exist between them:

$$
\mathbf{P} \cdot \mathbf{L}=0 \quad \mathbf{L}^{2}=a\left(1-\mathbf{P}^{2}\right)
$$

The collisions give rise to an additional mass flux in the stream. If $\delta f(\mathbf{r}, \mathbf{v}, t)$ designates the deviation of the distribution function from the initial distribution, this flux is given by

$$
\delta(n, \mathrm{U})=\int \mathbf{v} \delta f(\mathbf{r}, \mathbf{v}, t) d \mathbf{v}
$$

which can be shown to equal

$$
\delta(n, \mathbf{U})=-1 / 2 t^{2} \frac{\partial}{\partial \mathbf{r}} \cdot \Phi+O\left(t^{3}\right)
$$

where

$$
\begin{aligned}
\Phi & =\int \mathbf{w} C[F, F] d \mathbf{v} \\
& =\frac{\beta}{48} \pi D^{2} \int g\left[\beta g^{2} 1+3(\beta-4) \mathrm{gg}\right] f(\mathbf{v}) f\left(\mathbf{v}^{\prime}\right) d \mathbf{v} d \mathbf{v}^{\prime}
\end{aligned}
$$

with $\mathbf{g}=\mathbf{v}^{\prime}-\mathbf{v}$. 


\section{NUMERICAL RESULTS AND DISCUSSION}

For the special case of an azimuthally symmetric stream, the sixdimensional velocity integration in equation (13) was evaluated numerically. For this case, $F$ does depend only on $a, \mathbf{P}^{2}$, and $L_{z}$; and it is enough to study the situation in one cross section of the stream. Instead of $a, \mathrm{P}^{2}$, and $L_{z}$, the distribution function could also be expressed in terms of $a, e^{2}=\mathbf{P}^{2}$, and $\cos i$, where $i$ is inclination and $e$ eccentricity.

The results for a series of distribution functions of the type

$$
F\left(a, e^{2}, \cos i\right) \propto\left(a_{2}-a\right)\left(a-a_{1}\right)\left(e_{0}^{2}-e^{2}\right)\left(\cos ^{2} i-\cos ^{2} i_{0}\right)
$$

for $a_{1}<a<a_{2}, e<e_{0}$, and $i<i_{0}$ are presented below. The values for the parameters $a_{1}, a_{2}, e_{0}$, and $i_{0}$ were chosen to be

and

$$
a_{1}=0.8 \quad a_{2}=1.2
$$

$$
\begin{array}{lll}
\text { Case I: } & e_{0}=0.35 & i_{0}=7^{\circ} \\
\text { Case II: } & e_{0}=0.35 & i_{0}=13^{\circ} \\
\text { Case III: } & e_{0}=0.20 & i_{0}=13^{\circ} \\
\text { Case IV: } & e_{0}=0.10 & i_{0}=13^{\circ}
\end{array}
$$

The distribution functions were normalized such that the number of particles in the stream are the same for each case.

In figure 2 the density profile corresponding to case I along a radius in the equatorial plane of the stream is plotted. The shape of the profile is the same for the other cases except that it gets narrower with decreasing value of the maximum eccentricity $e_{0}$.

The kinetic pressure tensor takes a diagonal form in a spherical coordinate system. In figure 3, the three pressure components over density are plotted. The pressure in the azimuthal direction $P_{\phi \phi}$ is less than the two transverse pressure components. This is a general result, valid for all distribution functions that have been studied. By varying the maximal eccentricity and inclination, the ratio of the two transverse pressure components can be varied.

A general property of the collisions is to try to make the pressure tensor more isotropic. This gives rise to a most unusual property of the jetstream configuration that can be seen in figure 4 . Here the mass flux vector $\delta(n, \mathrm{U})$ is plotted for two points in a cross section of the stream, at $r=0.9$ and $r=1.1$ and at the same distance above the equatorial plane, represented by the broken line. The flux vector is plotted for three different values of the restitution parameter $\beta$ : $\beta=2$, which is the elastic case; $\beta=1$, which is the opposite extreme; and $\beta=1.5$, the intermediate value. The $x$ 's indicate the density maximum in the stream.

Consider the extreme cases I and IV, the former characterized by an excess pressure in the radial direction, the latter by an excess pressure in the polar 


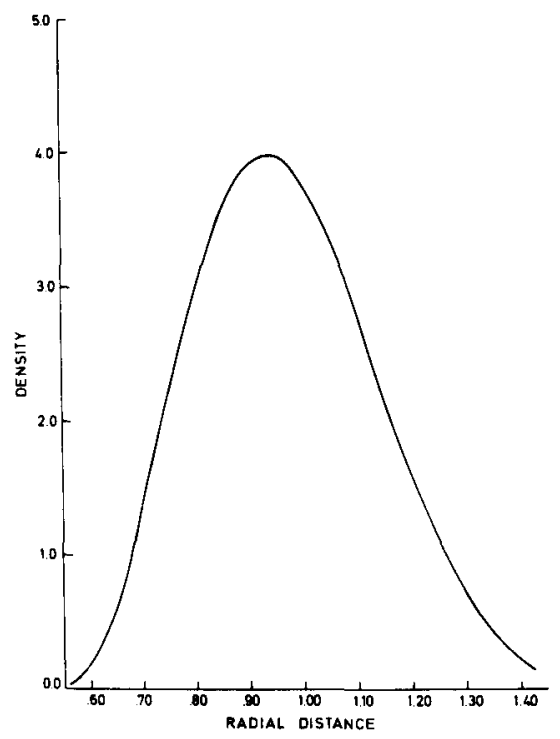

Figure 2.-Particle density in the stream along a radius vector in the symmetry plane of the stream, case I; arbitrary units.

direction. Common physical experience tells that if a system wants to eliminate excess pressure, it has to expand in some way. This is a statement of the Boyle-Marriotte ideal gas law. The jetstream behaves in the opposite manner. It eliminates excess pressure by contracting.

This can be understood from a consideration of the individual orbits in the stream. The central force field has the property of twisting these orbits very effectively. A particle that is on the bottom of the stream at one side will be on the top on the other side. A particle in a high-eccentricity orbit that is on the inner side of the stream at one time will be on the outer side half a period later. The pressure component in the polar direction is generated mainly by the orbits having the highest inclination. If the stream expands in this direction, this means that the distribution of inclinations must get wider. This again implies that the polar pressure increases. Thus for case I, to take care of the deficit in the polar pressure, the stream expands in this direction, contracting in the radial direction. Case IV represents the opposite case. Here the stream expands in the radial direction while contracting in the polar direction to eliminate excess pressure in the polar direction. Note that whereas an expansion or contraction in the polar direction is mainly an effect in inclination alone, the similar process in the radial direction is a more complicated phenomenon depending on the distribution of both eccentricity and the semimajor axis.

The effect of inelasticity always is to make the stream approach a more narrow configuration relative to the corresponding elastic case. This is in 

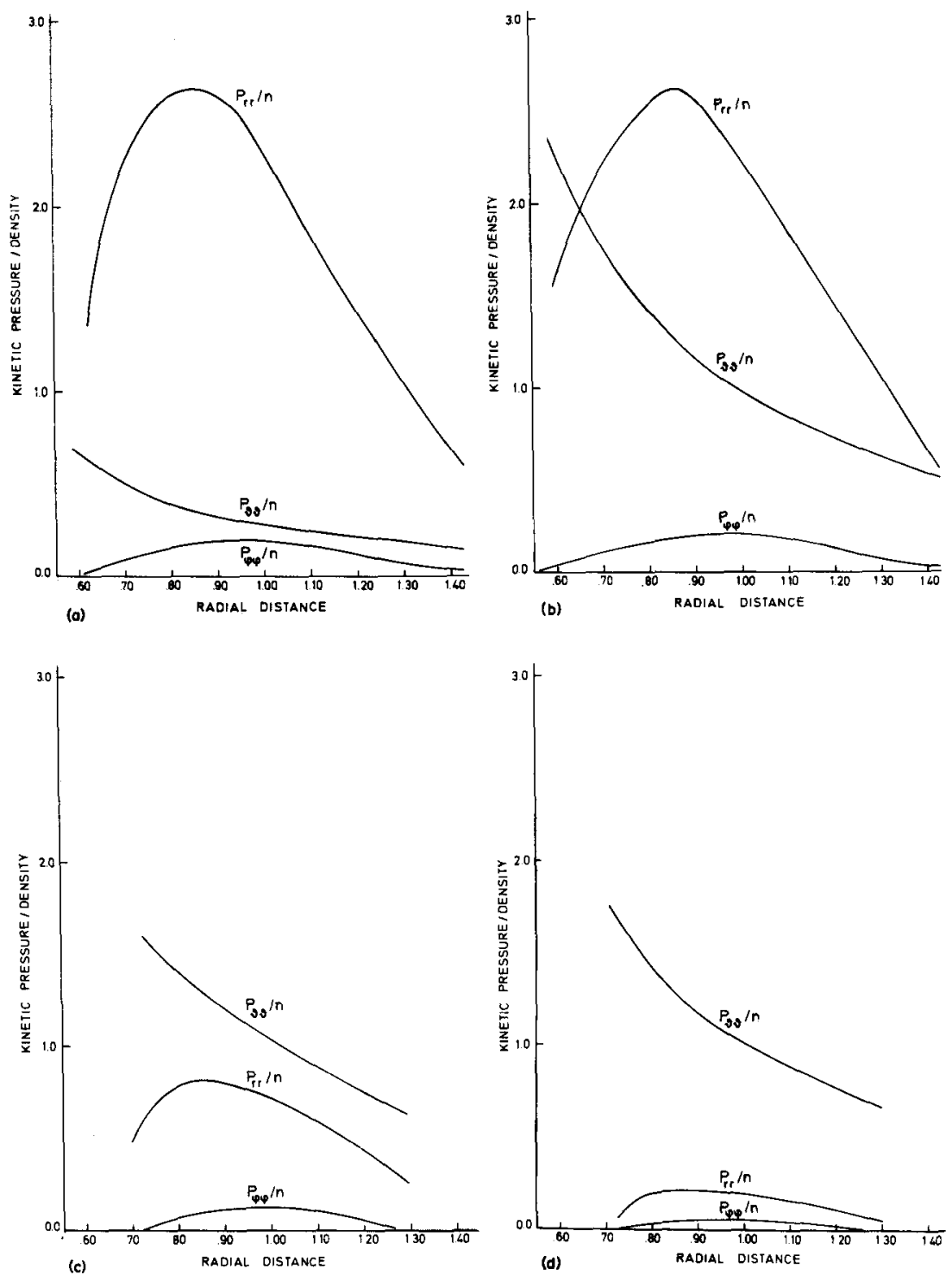

Figure 3.-Ratio of the three pressure components over density along a radius vector in the symmetry plane of the stream. (a) Case I. (b) Case II. (c) Case III. (d) Case IV. The same arbitrary units are used for all cases.

accordance with qualitative arguments. The degree of inelasticity, however, will have a varying degree of importance depending on details of the distribution function. For certain situations this parameter will determine whether the stream will expand or contract. Case II is such an example. 

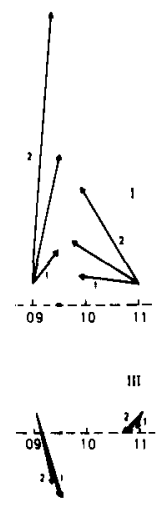

11

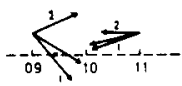

IV

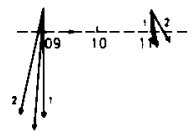

Figure 4.-Collision-induced mass flux at two points in a cross section of the stream for the four different cases studied. The broken line indicates the symmetry plane; the $x$ 's indicate the density maximum in the stream. The flux vectors are drawn for the three values $1.0,1.5$, and 2.0 of the restitution parameter $\beta$. The vectors belonging to the two extreme cases are indicated by 1 and 2 , respectively.

To estimate the time interval for which this type of analysis is valid, the ratio of the average mean free time between collisions to the average orbital period for the particles in the stream must be evaluated. This ratio is not a simple relation between particle diameter, mass, and volume of the stream, for instance. To a large degree it depends on the details of the distribution function. It is clear that this ratio increases rapidly as the particle diameter increases if the mass in the stream and the form of the distribution function are kept constant. According to the arguments presented above, this ratio will also increase with time, reducing the importance of collisions.

Results from an initial rate of change study should be interpreted with care because transient effects from a specific choice of initial state could easily mask important properties of the system. For our case, the above pattern was repeated for all distribution functions studied. Numerous questions, however, were not treated. Can instabilities develop in the stream? Is there a preferred profile the stream will try to reach? To what degree will the final state depend on initial state and on the degree of inelasticity? Answers to several of these and similar questions will hopefully be obtained from numerical simulations of jetstreams in the near future.

\section{REFERENCES}

Alfvén, H., and Arrhenius, G. 1970, Origin and Evolution of the Solar System, I. Astrophys. Space Sci. 8, 338.

Chapman, S., and Cowling, T. G. 1960, The Mathematical Theory of Non-Uniform Gases. Cambridge Univ. Press. London.

Danby, J. M. A. 1962, Fundamentals of Celestial Mechanics. Macmillan Co. New York. 